\title{
Commentary \\ The propofol infusion syndrome: more puzzling evidence on a complex and poorly characterized disorder
}

\author{
Olaf L Cremer
}

Department of Intensive Care, University Medical Centre Utrecht, Heidelberglaan 100, 3584 CX Utrecht, The Netherlands

Corresponding author: Olaf L Cremer, o.l.cremer@umcutrecht.nl

Published: 7 December 2009

Critical Care 2009, 13:1012 (doi:10.1186/cc8177)

This article is online at http://ccforum.com/content/13/6/1012

(c) 2009 BioMed Central Ltd

See related research by Roberts et al., http://ccforum.com/content/13/5/R169

\begin{abstract}
The propofol infusion syndrome is a potentially devastating cardiovascular and metabolic derangement that has been described in both pediatric and adult patients sedated with propofol. Despite a large number of case reports that have appeared in the literature since 1992, the precise clinical features and pathophysiology of this disorder remain uncertain. Historically, the syndrome has been characterized by the occurrence of lactic acidosis, rhabdomyolysis, and circulatory collapse after several days of high-dose propofol infusion. The affected patients were typically young and critically ill, and the reported mortality was high. More recently, a number of atypical cases have been reported with favorable outcomes. These occurred after short-term or lower-dose infusions in noncritically ill patients in whom generally only a subset of the classical syndrome features was observed. It remains unclear whether these reports reflect true propofol infusion syndrome detected at an earlier and more salvageable stage, or mere associations with the use of sedative agents in general. Without better information on the true incidence of the propofol infusion syndrome, clinical guidelines on the safe use of this drug remain unsupported by good evidence.
\end{abstract}

Since the propofol infusion syndrome (PRIS) was first described in 1992, clinical awareness and research interest into this disorder has continued to grow. The medical literature now includes over 100 published case reports, case series, letters, reviews, and occasional experiments. Nonetheless, the incidence and etiology of PRIS remain uncertain.

In the previous issue of Critical Care, Roberts and collaborators present the results of a multicenter study in which they prospectively measured the incidence of PRIS [1]. The authors monitored daily the clinical records of 1,017 critically ill patients who received propofol for nonprocedural sedation in the intensive care unit, and found that the combined occurrence of metabolic acidosis, cardiac dysfunction, and renal failure had an incidence of $1.1 \%$ in their study cohort.
This finding is puzzling for at least two reasons. First of all, because the clinical symptoms and excellent outcomes observed in the affected patients are at odds with most previously published cases. Secondly, because a dosedependent association between propofol exposure and the occurrence of these symptoms was absent.

When PRIS was first described, the symptoms that were reported consisted of bradyarrythmia, metabolic acidosis, cardiac failure, and death [2]. With time, the definition evolved to include rhabdomyolysis, hyperkalemia, hyperlipidemia, and renal failure, as well as specific electrocardiographic abnormalities in the right precordial leads resembling the Brugada pattern [3-5]. Although the precise causes of PRIS are unknown, there is both clinical and experimental evidence to suggest that propofol can trigger dysfunction of the mitochondrial respiratory chain, leading to depletion of ATP production and cellular hypoxia in tissues such as the heart and muscle [6]. Muscle biopsies and fat metabolism analyses of patients with PRIS resemble those found in mitochondrial cytopathies and acquired acyl-carnitine metabolism deficiencies by inhibition of beta oxidation $[7,8]$. The resulting accumulation of free fatty acids is a risk factor for cardiac dysrhythmia. These derangements may be triggered by or aggravated by metabolic stress and high energy demand (such as during critical illness), low carbohydrate supply (such as in children, due to their lower glycogen storage capacity), and high availability of fats (such as supplied by propofol's lipid emulsion) [9].

A major problem in studying PRIS is that many of its symptoms are nonspecific and common in critical care populations. Rhabdomyolysis, in contrast, occurs rarely in nontrauma patients, has previously been reported in over $50 \%$ of suspected PRIS cases, and can be directly linked to

PRIS = propofol infusion syndrome. 
the underlying mechanism of impaired muscular and myocardial free fatty acid utilization [10]. It is therefore remarkabe that rhabdomyolysis was not observed in a single case in the study by Roberts and colleagues. Brugada-like electrocardiographic abnormalities and hyperlipidemia also seem to be more specific findings in PRIS [4], but were likewise not observed. Because diagnostic tests other than those which occurred as part of routine clinical practice were not mandated in Roberts and colleagues' study, it is possible that these derangements were overlooked to some extent. Alternatively, the absence of these specific findings may indicate that the observed derangements were actually unrelated to propofol use.

PRIS has previously been rarely identified in prospective studies of propofol sedation in both children and adults, possibly because investigators have restricted propofol use to lower doses than those documented in most case reports [5]. In contrast, two retrospective cohort studies found an incidence of 6 to $10 \%$ in severe head trauma patients $[11,12]$. Although these patients were probably at increased baseline risk of developing this complication due to the nature of their neurological injuries, high metabolic stress, and the liberal use of vasopressors to maintain cerebral perfusion pressure, all cases had received high doses of the drug $(>83 \mu \mathrm{g} / \mathrm{kg} /$ minute) for prolonged periods of time (typically 4 days). Wysowski and Pollock analyzed the US Food and Drug Administration's Adverse Event Reporting System database and identified 68 adult patients who died after administration of propofol for nonprocedural sedation, $27(40 \%)$ of whom had symptoms consistent with the syndrome [13]. The median propofol dose in these patients was $90 \mu \mathrm{g} / \mathrm{kg} /$ minute and the median duration of use was 4.4 days.

Although there have recently appeared reports of patients developing metabolic acidosis as a possible early symptom of PRIS even during short-term infusions for general anesthesia [14], most studies in the medical literature suggest that the administration of propofol in higher doses and usually for longer durations greatly increases the risk of PRIS in both children and adults. It is conceivable that Roberts and colleagues failed to detect a dose-response relation because the exposure variation in their cohort was very limited by the fact that 10 of the 11 institutions who participated in the study had guidelines in place that restricted the use of propofol to a maximum of 60 to $83 \mu \mathrm{g} / \mathrm{kg} /$ minute - infusion rates that are currently considered safe for sedation in the intensive care unit.

To summarize, Roberts and colleagues are the first investigators to have systematically studied both the prevalence and incidence of PRIS manifestations in a prospective manner $[1,10]$. Their present findings challenge the current general concept that characterizes PRIS as a 'rare but lethal complication of prolonged high-dose propofol use'. Unfortunately, from their studies it remains uncertain whether all observed derangements truly reflect PRIS-related manifestations. To resolve this issue, future prospective studies will need to observe matched cohorts of critically ill patients exposed to various sedation regimens. According to the authors' estimate, such a comparison would require at least 2,000 patients per arm. Until such data become available, clinical guidance on the safe use of propofol in the intensive care unit remains unsupported by evidence.

\section{Competing interests}

The author declares that they have no competing interests.

\section{References}

1. Roberts RJ, Barletta JF, Fong JJ, Schumaker G, Kuper PJ, Papadopoulos S, Yogaratnam D, Kendall E, Xamplas R, Gerlach AT, Szumita P, Anger K, Arpino P, Voils S, Grgurich P, Ruthazer $\mathrm{R}$, Devlin JW: Incidence of propofol-related infusion syndrome in critically ill adults: a prospective, multicenter study. Crit Care 2009, 13:R169.

2. Parke TJ, Stevens JE, Rice AS, Greenaway CL, Bray RJ, Smith PJ, Waldmann CS, Verghese C: Metabolic acidosis and fatal myocardial failure after propofol infusion in children: five case reports. Br Med J 1992, 305:613-616.

3. Fudickar A, Bein B: Propofol infusion syndrome: update of clinical manifestation and pathofysiology. Minerva Anestesiol 2009, 75:339-344.

4. Vernooy K, Delhaas T, Cremer OL, Di Diego JM, Oliva A, Timmermans C, Volders PG, Prinzen FW, Crijns HJ, Antzelevitch C, Kalkman CJ, Rodriguez LM, Brugada R: Electrocardiographic changes predicting sudden death in propofol-related infusion syndrome. Heart Rhythm 2006, 3:131-137.

5. Ahlen K, Buckley CJ, Goodale DB, Pulsford AH: The 'propofol infusion syndrome': the facts, their interpretation and implications for patient care. Eur J Anaesthesio/ 2006, 23:990-998.

6. Ypsilantis P, Politou M, Mikroulis D, Pitiakoudis M, Lambropoulou M, Tsigalou C, Didilis V, Bougioukas G, Papadopoulos N, Manolas C, Simopoulos C: Organ toxicity and mortality in propofol-sedated rabbits under prolonged mechanical ventilation. Anesth Analg 2007, 105:155-166.

7. Wolf $A$, Weir $P$, Segar $P$, Stone J, Shield J: Impaired fatty acid oxidation in propofol infusion syndrome. Lancet 2001, 357: 606-607.

8. Withington DE, Decell MK, Al Ayed T: A case of propofol toxicity: further evidence for a causal mechanism. Paediatr Anaesth 2004, 14:435-438.

9. Otterspoor LC, Kalkman CJ, Cremer OL: Update on the propofol infusion syndrome in ICU management of patients with head injury. Curr Opin Anaesthesiol 2008, 21:544-551.

10. Fong JJ, Sylvia L, Ruthazer R, Schumaker G, Kcomt M, Devlin JW: Predictors of mortality in patients with suspected propofol infusion syndrome. Crit Care Med 2008, 36:2281-2287.

11. Cremer OL, Moons KG, Bouman EA, Kruijswijk JE, de Smet AM, Kalkman CJ: Long-term propofol infusion and cardiac failure in adult head-injured patients. Lancet 2001, 357:117118.

12. Smith H, Sinson G, Varelas P: Vasopressors and propofol infusion syndrome in severe head trauma. Neurocrit Care 2009, 10:166-172.

13. Wysowski DK, Pollock ML: Reports of death with use of propofol (Diprivan) for nonprocedural (long-term) sedation and literature review. Anesthesiology 2006, 105:1047-1051.

14. Cravens GT, Packer DL, Johnson ME: Incidence of propofol infusion syndrome during noninvasive radiofrequency ablation for atrial flutter or fibrillation. Anesthesiology 2007, 106: 1134-1138. 